\title{
Géométrie des interfaces et niveaux énergétiques ; modalités d'étude d'une interface morphologique : le Lodévois (Hérault, France)
}

\author{
Ph. Martin \\ Université d'Avignon et des Pays de Vaucluse, UFR-ip SHS, UMR ESPACE 7300 du CNRS, 74 rue L. Pasteur, \\ case 19, 84029 Avignon Cedex, France
}

\begin{abstract}
Résumé. Nous présentons les conditions épistémologiques d'une étude des structures scalaires des interfaces. Les cas non géographiques sont définis (morphologie euclidienne, surface brownienne). Ce sont des morphologies possédant des degrés élevés de symétrie. La matérialité de ces formes conditionne aussi leur imperfection. Les structurations scalaires sont complexes lorsque les entités considérées sont soumises à des contraintes (spatiales ou autres) et à des flux importants, surtout si les réactions qui se réalisent sur les interfaces sont lentes. Un tableau général qui croise les niveaux énergétiques, les morphogenèses, les symétries, les structures scalaires, les invariants et les règles structurelles (attracteurs) recherchés est proposé. Un exemple d'application est développé (Lodévois, France). Elle utilise une extraction originale de l'information (Coupe cordée Topographique ; CcT : simple, ou en miroir ${ }^{M} \mathrm{CcT}$ ), la méthode dite RMS de calcul de l'exposant $\mathrm{H}$ de Hurst et une analyse de la variation locale de $\mathrm{H}$ en fonction des échelles. Des rapports encore hypothétiques sont faits avec les résultats déductifs issus de la Relativité d'échelle de L. Nottale [1] qui sert de cadre à cette réflexion.
\end{abstract}

Si sur les interfaces se réalisent les réactions, des interactions, des dynamiques qui animent les systèmes ou les entités en présence, celles-ci ne peuvent s'effectuer sans que ces lieux d'échanges et de confrontations aient une géométrie qui est, en outre, particulière en fonction de certains critères comme le rapport entre l'intensité des flux (massique, d'énergie et d'information) et les vitesses, les cinétiques des réactions, des transformations réalisées à la plus grande échelle. Autrement dit si les réactions sont lentes et les flux massiques nécessaires importants pour atteindre un but quel qu'il soit (maintien de la vie, érosion rapide d'un relief, etc.) il est nécessaire que la surface sur laquelle se font les échanges acquière des caractéristiques particulières qui traduisent une organisation scalaire de l'entité.

Se dessinent ainsi deux types de formes pour les interfaces avec, d'une part, celles qui correspondent à des dynamiques faibles ou lentes et dans ce cas c'est la géométrie euclidienne qui constitue la base des archétypes morphologiques en jeu (surfaces lisses, formes régulières, etc.), - les réactions elles se faisant globalement sur un mode linéaire (proportionnalité des causes et des conséquences) et, d'autre part, celles pour lesquelles la dynamique est intense ou aiguë (croissance forte, érosion forte, etc.) et dans ce cas, la géométrie sous-jacente est celle de la fractalité qui se cristallise dans des structures spatiales modélisables (surfaces rugueuses, tourmentées, formes apparemment irrégulières, etc.) ; la différence fondamentale entre ces

a e-mail : philippe.martin@univ-avignon.fr deux régimes (et ces apparences morphologiques) étant dans le rôle que jouent les échelles dans ces situations. Dans le premier cas elles ne jouent presque aucun rôle - nous sommes alors en situation d'indépendance d'échelle - dans le second elles interfèrent fortement et nous sommes alors en situation de dépendance d'échelle. Si l'indépendance d'échelle est une catégorie générique sans modalités particulières, il n'en est pas de même pour la dépendance qui se décline en invariance d'échelle (dimension fractale $\mathrm{D}^{\mathrm{F}}$ - constante), en transitions Fractal - Non-Fractal (F-NF, ou NF-F ou NF-F-NF ou F-NF-F) qui correspondent aux passages, pour un même espace ou entité, d'une situation de dépendance d'échelle $(F)$ à une situation d'indépendance d'échelle (NF), etc., mais aussi en dimension fractale variable, etc. En d'autres termes, la structuration scalaire (loi d'échelle) dont la manifestation à la limite est une fractale peut être très variable et ne concerner qu'une portion de la gamme scalaire intéressant l'espace ou l'entité considérée. Ces lois (du moins les plus simples) ont aujourd'hui été formalisées de manière purement déductive dans une théorie développée par L. Nottale [1,2] dans le cadre de la Relativité d'Échelle (RE) à laquelle nous renvoyons le lecteur. Dans l'approche ici proposée nous montrerons graphiquement que nous retrouvons certaines de ces lois d'échelle dans l'analyse d'un relief : le Lodévois (France) situé entre la retombée méridionale du Larzac et la plaine de l'Hérault, mais qu'il conviendrait aussi, vraisemblablement, de leur adjoindre une modalité supplémentaire afin de tenir compte de transitions F-NF moins simples qu'analytiquement initialement envisagé. Pour arriver à ce résultat nous mettrons en œuvre une méthode d'extraction et de mise en forme de l'information 
utilisée originale, car inventée spécifiquement pour essayer de faire entrer la géomorphologie dans un cadre théorique d'essence géométrique.

\section{Symétries et objets géographiques}

Les objets géographiques (et donc leurs formes), autrement dit les entités isolables déployées à des échelles comprises entre $10^{-4} \mathrm{~m}$ et $10^{+8} \mathrm{~m}$ environ au niveau de l'interface terrestre, pour l'essentiel, que nous peuplons, peuvent être envisagés au travers des symétries classiques (translation, rotation, miroir) et moins habituelles (dilatation - contraction). Dans un cas, ces entités sont euclidiennes ; dans l'autre, elles sont fractales. Toutefois, ne sont géographiques, comme nous allons le voir ci-dessous, que des entités qui ne sont pas totalement symétriques dans la mesure où toute morphogenèse, toute apparition d'une nouvelle forme correspondent à une brisure de symétrie. Or, cette forme (variation du nombre, du degré de symétrie) renvoie dans l'espace à une structure, c'est-à-dire à une loi capable d'encoder spatialement une information non triviale ; loi qu'il convient d'établir.

\subsection{Des entités a-géographiques car totalement symétriques}

Pour arriver à penser des objets géographiques dans un cadre théorique et quantifié il faut remarquer qu'ils constituent une classe d'entités bornée par deux situations en partie antithétiques [3-6]. D'un côté, nous trouvons une configuration spatiale parfaitement monoscalaire où toute occupation d'un lieu par un attribut est équivalente à toute autre. L'image qui permet peut-être de penser cela est une mer d'huile ; un espace d'une planéité totale où tout centimètre carré est égal à tout autre (homogénéité des caractéristiques de l'eau de mer) dans n'importe quelles directions que ce soit et à n'importe quelles distances que ce soit. Par conséquent, il n'y a aucune différence après application de symétries (translation, rotation, miroir, dilatation). Ce milieu est totalement symétrique et on pourrait parfaire l'illustration en introduisant la profondeur. Si l'entité peut avoir une forme (surface), elle n'a pas de structure scalaire, donc sa dimension est égale à deux $(\mathrm{D}=2)$ ou à trois si on considère le volume $(X, Y, Z)$. Cette surface miroitante est une forme euclidienne pour laquelle la distribution de l'attribut (eau de mer) est totalement déterministe. Sachant ce qu'il y a là, je peux en déduire ce qu'il y a là-bas à n'importe quelles distances et dans n'importe quelles orientations choisies. Sans apports d'énergie qui vienne troubler cette surface (risée produite par un souffle de vent), celle-ci peut rester ainsi un temps infini. Elle est donc dans un état stable au sens thermodynamique. Cette situation archétypale a un pendant sur les continents au travers de ce que les géomorphologues appellent une surface d'aplanissement, même si celles-ci ne sont pas aussi parfaites et homogènes qu'une surface miroitante d'eau. Il faut bien voir que cet archétype prend en défaut les questionnements fondamentaux de la géographie qui visent à faire émerger une compréhension des différences de distribution dans l'espace des attributs. De ce point de vue cette forme est a-géographique, mais il y en a une autre qui l'est tout autant en n'ayant absolument pas la même morphologie. C'est la surface d'une mer déchainée, d'une mer en furie lors d'une tempête. Sur le continent, on peut penser comme pendant aux Tsingy malgaches. Dans ce type de morphologie, il y a certes des creux et des bosses, des pointes et des gouffres, mais ceux-ci sont totalement dus au hasard. Ces surfaces illustrent un autre archétype que l'on appelle une surface brownienne. Certes, celle-ci est structurée dans les échelles puisqu'il y a des petites, des moyennes ou des grandes bosses (tout autant que des petits, des moyens ou des grands creux), mais cette organisation n'est que le produit du hasard, dans la mesure où une surface brownienne est l'extension en trois dimensions du mouvement brownien dont tant la direction que la longueur des pas successifs sont choisies au hasard dans le cadre d'une distribution globalement gaussienne. Autrement dit, tout kilomètre carré d'une mer en furie sera égal à toutes dizaines de kilomètres carrés ou à tout hectare selon une parfaite symétrie par dilatation ou contraction. Ces caractéristiques font que le fait de connaitre la position d'un point (dans un creux ou sur une bosse) ne renseigne en rien sur la position de tout autre point dans n'importe quelles directions que ce soit et à n'importe quelles distances que ce soit. Il n'y existe donc aucune autocorrélation spatiale, donc toute analyse spatiale est impossible. Il n'y a ainsi aucune logique d'ordre spécifique dans les dimensions de cet objet $(X, Y, \varepsilon)$. Toutefois, cette structure scalaire peut être mesurée. Elle est égale à un plus la dimension fractale de mouvement brownien soit $1+1,5=2,5$ et l'exposant $\mathrm{H}$ de Hurst y est égal à 0,5 pour toutes les coupes. Aucune étude fondée sur les questionnements de base de la géographie n'est possible dans ce cas. L'indétermination est totale. La seule connaissance que nous pouvons en avoir serait d'enregistrer tous les points de cette surface, ce qui serait sans intérêt puisqu'elle aurait les mêmes caractéristiques que tout autre, construite sur la même base aléatoire, et cela même si la seconde réalisée n'avait pas exactement la même morphologie apparente que la première calculée. Ceci montre en outre qu'il y a un écart entre la perception que nous pouvons avoir des morphologies et les résultats des mesures que nous pouvons en faire, comme nous le verrons ci-dessous, ce qui conduit à relativiser l'approche phénoménologique. Même si les mots pour dire la surface parfaite de la mer d'huile sont plus nombreux que ceux pour évoquer la mer en furie, nous sentons bien que peu de choses sont dicibles à partir de la perception simplement parce que peu de choses sont perçues comme différenciées.

Sur ces bases, il est une autre opposition didactique que nous devons introduire afin de mieux penser ce que sont les différences des interfaces considérées statiquement.

\subsection{Entités, géométries et types de rapports scalaires}

Dans le cas de la mer d'huile, nous sommes face à une entité qui appartient à une classe d'objets archétypaux conceptuels dans laquelle on retrouve tant la courbe de flambage pour les méandres, que l'idée de la sphère pour la Terre ou encore celle du parallélépipède rectangle pour les bâtiments. Cela correspond à des entités (objets - idées) euclidiennes et nous sommes alors dans une situation d'indépendance d'échelle. En d'autres termes, la variation de la courbure 
sera aussi homogène que possible en tous points de la courbe de flambage, tout comme dans le cas de coupes réalisées à la surface d'une sphère (cercle), ce qui permet de les classer dans les courbes différentiables (par tout point, il ne peut passer qu'une seule tangente). En outre, toute portion de sphère sera semblable à toute autre, située ailleurs. Cela traduit l'expression de symétries (translation, rotation, miroir). Lesquelles, comme nous allons le voir dans le second cas, peuvent être complétées par une symétrie d'échelle, qui peut mettre en jeu, comme forme de base, de tels objets (cubes, etc.) dans une structure homothétique. Dans ce dernier cas, les observations, les situations, les configurations sont homologues entre deux niveaux à un facteur d'échelle près. Les niveaux étant définis par la taille de l'objet initial et par le facteur de réduction ou de dilatation utilisé.

Dans le second cas (mer en furie cf. ci-dessus), nous sommes en situation de dépendance d'échelle ce qui signifie que les configurations géométriques (les morphologies constatées, mais aussi les dynamiques qui leur ont donné naissance) varient en fonction de l'échelle à laquelle on les considère. Toutefois, la forme répétée dans les échelles n'est que statistiquement semblable. C'est le cas général regroupé sous le vocable de fractalité. Dans cette situation, l'information varie selon l'échelle, ce dont rend compte la dimension fractale qui quantifie son taux scalairement local (entre deux échelles) de variation, celui-ci pouvant être constant (situation d'invariance d'échelle) ou variable (situation de transition et/ou de covariance : dimension fractale variable, $[1,2]$. Bien que la conceptualisation de cette organisation scalaire soit récente [7,8], l'ubiquité de ce mode de structuration est aujourd'hui évidente tant pour des objets ou des entités naturels (montagne, nuage, etc.) que pour des constructions ou des réalisations anthropiques d'une certaine ampleur (ville, frontière, réseau, etc.). Dans ces cas, le fait d'introduire l'équivalent d'une homothétie pour mieux coloniser l'espace, donc pour la ville des parallélépipèdes rectangles grands, moyens ou petits, fait basculer l'entité dans le champ de la fractalité sans que cela ait été ni perçu avant le milieu des années soixante, ni évidemment voulu. Il s'agit au fond d'une solution d'optimisation qui met en œuvre les échelles [9] et celleci est d'autant plus forte que la possibilité - lorsque l'entité est poussée à croître - d'utiliser un déploiement spatial (plus ou moins en nappe) est faible. Les villes occidentales en particulier portent les stigmates d'une possibilité de déplacement automobile et d'une abondance quasi illimitée à très bas coût d'énergie. Lorsqu'à l'inverse l'extension n'est plus possible, pour des raisons économiques (prix de l'énergie), techniques (dégradation des solutions techniques sophistiquées), politiques (contrôle accru des populations), culturelles (repli sur un isolat), etc. alors on aboutit à des formes urbaines comme les médinas dont il faudrait établir la structure scalaire à partir d'informations en trois dimensions. Ces deux possibilités (étalement, densification scalaire) sont donc conjoncturelles et il y a certainement en fonction du système technique, culturel, économique, etc. à l'œuvre une structuration scalo-spatiale optimale qu'il faudrait rechercher [5]. On voit au travers de ces remarques que la ville est une interface beaucoup plus complexe que celles dont nous allons traiter ci-dessous, mais qui peuvent servir de modèle.
Sur ces bases, il faut en outre introduire deux oppositions. L'une concerne la différence existant entre les archétypes conceptuels (la sphère pour reprendre l'un des exemples donnés ci-dessus, mais aussi comme la courbe de Von Koch ou de Peano pour citer des cas fractals fractales déterministes) et les matérialisations qui peuvent exister : le cerceau pour le cercle et le dessin de la courbe de Von Koch pour cette dernière. Dans le cas de l'archétype conceptuel, nous sommes dans un cadre idéel (platonicien) correspondant à une pure information qui peut être exprimée et travaillée à partir d'un pur formalisme mathématique. Les propriétés démontrées sont alors absolument vraies comme la différentiabilité qui peut s'entrevoir comme étant au plan tactile la manifestation du lisse absolu, de la régularité totale (mais aussi dynamique en tendance et/ou cyclique). Dans l'autre, nous sommes dans le domaine matériel, dans celui de la cristallisation, avec ses imperfections et ses limites, de l'archétype conceptuel. Un cerceau c'est (presque) un cercle, sauf qu'il a une épaisseur, une rugosité, de légères déformations, etc. Dans ce cas, les propriétés sont celles formalisées dans le cadre euclidien... à la limite, in abstracto, en faisant abstraction des imperfections ou des impératifs de construction. En d'autres termes, l'objet (au sens constructiviste du terme : celui que l'on peut construire de nos mains) euclidien n'existe pas ; il n'en existe que des réalisations qui s'approchent plus ou moins du concept, ce qui limite d'ailleurs fortement dans leur portée et intrinsèquement les épistémologies constructivistes souvent invoquées en géographie.

\subsection{Fractales déterministes et matérialité des structures fractales}

Or, il en est de même pour l'objet fractal dès que nous essayons de le construire dans la mesure où une fractale est une configuration, un état organisé particulier à la limite (au sens mathématique), quand l'on considère ce qui se passe lorsque l'on fait tendre les échelles mises en œuvre, soit vers la plus grande, soit vers la plus petite. En cela une fractale théorique (exprimée seulement par un code formel) est l'homologue d'un cercle euclidien, mais en intégrant une dimension d'échelle, ce que ne font pas les archétypes euclidiens, comme nous l'avons vu. Une fractale (déterministe) est donc aussi un archétype conceptuel, mais avec en outre une difficulté qui est que ces organisations sont - à la limite - non différentiables et donc que certains outils mathématiques classiques comme la différenciation ne leur sont pas, à la limite, applicables. Cela étant, rien n'implique d'aller jusque-là. Rien ne nous y oblige, d'autant que les entités matérielles que nous avons à considérer, ici une montagne, là une ville, n'ont pas une extension scalaire qui va jusqu'à l'échelle la plus grande ou la plus petite située au niveau de l'un des infinis $(+\infty$ ou $-\infty)$. Ceci explique pourquoi il est peu satisfaisant intellectuellement d'utiliser des fractales déterministes du type du tapis de Sierpinsky pour rendre compte (voire modéliser) par exemple la nappe du bâti urbain, sauf à introduire ex nihilo des limites scalaires difficiles à justifier.

En d'autres termes, les caractéristiques liées à la matérialité des entités, des interfaces qui nous occupent ici, comme le projet géographique qui situe les problèmes abordés par cette discipline entre $10^{-4} \mathrm{~m}$ et $10^{+8} \mathrm{~m}$, donc 
à des échelles périhumaines, nous autorisent à ne pas nous préoccuper de ce qui se passe à la limite, et au contraire de porter toute notre attention sur les configurations qui apparaissent dans l'entregent de leurs bornes scalaires, et cela même en utilisant les possibilités offertes par les équations différentielles comme l'a bien montré L. Nottale $[1,2]$.

Ainsi, tout comme un cerceau n'est pas un cercle, une forme tangible, perceptible «fractale» n'est pas une fractale. On voit dès lors que si le vocabulaire français possède deux mots pour dire ce qui relève d'une matérialité qui a été perçue et conçue en premier et ce qui relève de l'archétype conceptuel (ce qui a été établi postérieurement), il n'en possède qu'un pour dire ces organisations scalaires qui ont été nommées à partir de leur démonstration mathématique, en sautant donc l'étape de la reconnaissance phénoménologique, ce qui est d'autant plus vrai que chacun avait sous les yeux, depuis des siècles, des objets intégrant l'archétype fractal qui n'a pu être vu avant d'avoir été conçu. Ceci renvoie donc évidemment à une grande interrogation qui est de savoir pourquoi les formes déployées selon des archétypes euclidiens ont pu être très tôt reconnues et utilisées par l'humanité (pyramide, lettre des alphabets, centuriation romaine, etc.) alors que celles intégrant des archétypes fractals ne l'ont été que très tardivement et encore en passant par un formalisme, c'est-à-dire en se dotant d'une «prothèse », comme le microscope a pu en être une. Il est clair que la réponse se trouve dans nos caractéristiques neurophysiologiques, et il est tout à l'honneur du géographe de porter et d'apporter au monde un discours explicitant cette dimension de l'univers aux échelles anthropiques.

Ainsi, comme nous parlerons de cerceau et de cercle, de méandre et de courbe de flambage nous parlerons de scalarité pour dire l'organisation scalaire continue entre deux échelles, l'une grande, l'autre petite, délimitant, sur ce plan, l'entité considérée et nous utiliserons le terme de fractalité pour signifier l'état archétypal, à la limite, du modèle de cette entité lorsqu'il est codé soit sous une forme algorithmique, soit construit dans une expérience de pensée, donc hors de toute imperfection liée à la matérialité.

Reste une question qui est celle de la dynamique des interfaces au sens de leur morphogenèse. Celle-ci est sous la coupe du niveau énergétique, ce qui implique qu'un accroissement, en particulier des échanges énergétiques, mais aussi informationnels, impliquera une évolution vers une intégration plus importante de la dimension d'échelle et inversement. En d'autres termes, si la configuration fractale au sens d'un archétype défini à la limite peut être l'attracteur stable - la morphologie parfaite vers laquelle tend l'entité de la dynamique considérée, la configuration scalaire va être le mode d'organisation changeant, contraint par l'évolution du niveau énergétique, donc ce qui est à considérer au premier chef dans une modélisation.

\section{Morphologie, contrainte spatiale, intensité des flux et déploiement dans les échelles}

Or, qu'est-ce qui différencie une surface si ce n'est une morphologie : une vague, les ondes liées au plouf d'un poisson, etc. ? Celles-ci sont des formes qui viennent briser la symétrie de la forme initiale et introduire une structure spatiale qui peut elle-même être analysée (par exemple comme un système d'ondes concentriques dégradant l'énergie introduite dans le milieu liquide par le saut du poisson). Mais, le mécanisme peut fonctionner dans les deux sens énergétiques (apport ou dissipation). Le vent qui se lève produit la vaguelette, mais le vent qui se calme transforme la surface brownienne de l'océan en une suite de vagues, en une houle plus ou moins croisée, en des formes dicibles, car identifiables, car particulièrement structurées, donc non aléatoires, dans l'espace scalaire considéré. Les formes géographiques sont donc celles qui se situent entre ces deux extrêmes, bien plus objets de pensée qu'entités tangibles, et loin des systèmes soit sans aucune énergie, soit débordant d'énergie. Leur matérialité et leurs rapports à ce qui est autre les assignant à une gamme scalaire particulière. Il n'y a pas de cellules de la taille d'un ballon de baudruche (et inversement). Toute évolution, dans un sens ou dans un autre, se fait donc par une évolution du degré de symétrie (morphogenèse vers des formes plus homogènes ou plus aléatoires). Toutefois, dans les exemples illustratifs pris ci-dessus le milieu considéré (la mer) était soit tranquille, car ne recevant aucune énergie, soit agitée - au sens propre du terme - comme quelque chose qui serait secoué de l'extérieur, comme des arbres vibrés par un tremblement de terre. Autrement dit, ces morphologies ne sont pas particulièrement liées à des échanges.

\subsection{Cristallisation de rapports scalaires variables et cinétique des réactions}

En particulier dans les processus de croissance ou d'érosions (départ de matière) apparaissent des formes qui dépendent de critères d'optimisation comme la ville envisagée ci-dessus. On peut ainsi penser par exemple à toutes les situations végétales (arbres, etc.) ou animales (poumons, etc.) dans lesquelles les cinétiques des réactions nécessaires à la survie de l'entité sont lentes, mais pour lesquelles les flux nécessaires au maintien de la vie sont importants. Dans ces cas, les croissances observées et les formes atteintes présentent un facteur d'échelle et une structuration en échelle. C'est en particulier le cas lorsqu'il y a en outre une contrainte spatiale (place limitée comme dans une cage thoracique). Les reliefs en érosion correspondent en fait à une situation de réduction d'un écart à la situation stable qui est comme nous l'avons indiqué, la surface d'aplanissement. Il s'agit donc dans ces systèmes d'un processus de dégradation d'une énergie fournie par les réactions nucléaires du centre de la Terre via moult mouvements et manifestations possibles. Or, pour que la réduction d'un relief même important (tout apport énergétique ayant cessé) se fasse dans un temps géologique fini et aussi court que possible (donc conformément aux lois de la thermodynamique), il est nécessaire en fonction de la lenteur des processus physico-chimiques de dégradation, d'altération, de transport, etc. des roches que la surface exposée soit aussi vaste que possible [3,4,10-12]. Ceci est acquis essentiellement par l'action des rigoles, des ruisseaux, des rivières, des fleuves qui littéralement griffent (glyptogenèse) et creusent la surface saillante dès qu'elle se déploie sous la contrainte des forces tectoniques. Il est 
clair que si cette action d'érosion au sens strict pouvait être aussi rapide que les morphogenèses liées à la tectonique, aucun déploiement orographique ne pourrait avoir lieu. Ces dynamiques se différenciant d'environ un facteur 10 , il faut nécessairement que la tectogénèse s'arrête pour que le relief soit arasé, mais bien longtemps après. Or, les reliefs récents, comme d'ailleurs les très anciens présentent des morphologies qui sont très loin des plus emblématiques comme les Alpes, les Rocheuses ou l'Himalaya. Ces massifs possèdent des morphologies particulièrement découpées par l'ensemble des réseaux de drainage (et par d'autres processus) qui sont en accord avec le niveau énergétique observé, c'est-à-dire avec la grande énergie disponible grâce à l'élévation au-dessus du niveau de l'océan mondial, réalisée ; les montagnes étant en général arrosées par des pluies. Il apparait donc un lien entre les morphologies des massifs et leur niveau énergétique. S'il est impossible d'observer le déploiement orogénique et la réduction érosive d'un massif, il est par contre possible d'envisager dans une approche ergodique, $\mathrm{X}$ massifs présentant des morphologies différentes dont nous pouvons faire l'hypothèse qu'elles correspondent à des stades d'évolution particuliers entre la surface d'aplanissement originelle et le retour à un état aplani, de portions de la surface terrestre (cycle davisien). Clairement, nous retrouvons là, l'anthropomorphisme en moins, les idées de montagnes ; jeunes, mures, vielles, etc. qui structuraient les manuels de géographie physique il y a quelques dizaines d'années et que bien des écoliers ont apprises en comparant les Drus au ballon de Guebwiller. Il est donc clair que pour aller plus loin il est nécessaire de dépasser ce vocabulaire en nous dotant d'outils décrivant et quantifiant la structuration en particulier scalaire de ces surfaces, de ces interfaces géologiques. C'est ce que nous proposons de faire dans la troisième partie de ce texte.

Cela étant avant d'en arriver là il faut revenir

sur les relations qui existent entre les modes de dynamique, les niveaux énergétiques, les morphogénèses induites, la situation au regard des symétries, la désignation des structures scalaires, les types d'invariant et l'arrière-plan structurel que matérialise un attracteur. Tout cela a été mis en regard dans le tableau $\mathrm{n}^{\circ} 1$ qui livre donc d'une part les correspondances entre situations énergétiques, morphologies et structures scalaires et d'autre part le vocabulaire en particulier dans le champ des rapports scalaires, où il est encore très mouvant et imprécis dès que l'on est en situation de dépendance d'échelle, mais au-delà de l'invariance d'échelle ; ce qui a été codifié par L. Nottale [1,2] dans la théorie de la Relativité d'échelle.

On peut donc résumer ce qui précède en notant que les morphologies cristallisent les rapports existant entre les cinétiques des réactions se produisant aux interfaces et les flux dont toute entité a besoin pour perdurer (maintien d'un état stationnaire) ou se voit affublée pour un retour aussi rapide que possible vers un état stable (problème de l'érosion). Par ailleurs, le déploiement scalaire (ampleur de la gamme des échelles concernées, type de relations scalaires : $D_{F}$ unique, variable, etc.) est d'autant plus important et complexe (facteurs additionnels expliquant l'accélération d'échelle manifestée par une dimension fractale continument variable dans l'ordre des échelles) que la contrainte spatiale est forte et l'intensité des flux élevée.

Sur ces bases épistémologiques et problématiques, nous montrerons à partir d'un exemple pris en géographie physique comment il est possible d'analyser les morphologies de certaines de ces interfaces, et ce que l'on peut en attendre au plan conceptuel et pratique. Pour cela, nous nous inspirerons des travaux conduits sur des interfaces industrielles définies souvent à très haute résolution [13].

\section{Structuration scalaire du Lodévois}

Dans l'une de nos approches théoriques de reliefs à base géométrique, nous avons choisi de travailler sur le Lodévois [14] situé au sud du Massif Central (France), car il présente des reliefs importants (rebord sud du Larzac au nord, échine de l'Escandorgue à l'ouest, etc.) et des dépressions notables (du Salagou au sud-ouest, le piémont languedocien se raccordant à la vallée de l'Hérault au sud-est, etc.) à peu de distance les uns des autres. Certes au plan lithologique, stratigraphique, et si l'on regarde l'histoire géomorphologique de ces entités une grande variété apparait. Au karst de plateau nu est associée une forme volcanique qui domine un fossé tectonique permo-triasique et une plaine d'épandage largement néogène, et quaternaire pour les derniers termes. Cela étant, ces reliefs sont bien l'un à côté de l'autre et c'est cela que très largement l'analyse spatiale (mais aussi scalaire) développée en géographie essaye de caractériser, de comprendre et que l'approche fractale vise à modéliser dans sa dimension d'échelle.

Il existe comme justificatif de cette démarche un postulat, que l'on peut ou non partager, mais qui est le suivant. Certes, les roches sont d'âges très variables, certes, les phases orogéniques ont pu être très dissemblables (pensons par exemple à la régression messinienne et à la transgression pliocène, aux différentes positions de la Méditerranée au cours du Quaternaire, etc.), mais cela n'empêche pas que la morphologie observée et observable puisse être caractérisée en faisant fi de ces considérations. Ceci conduit donc à penser que les dynamiques qui se réalisent sur cette interface entre lithosphère et atmosphère sont infiniment plus importantes pour comprendre l'existant que la caractérisation des jalons chronologiques qui permettent de reconstituer l'histoire de cet espace, ou de tout autre. Pour qu'il en soit ainsi, donc pour que les déterminants lithologiques et paléogéographiques soient considérés comme mineurs, il faut que derrière la dynamique d'interface sur le temps long géologique que ces morphologies cristallisent, il y ait des déterminants structurels puissants. A contrario, si nous arrivons à mettre en évidence des lois scalaires organisant ces paysages cela tendra à indiquer que ces déterminants structurels sont bien présents. De plus si les caractérisations auxquelles nous pouvons aboutir sont conformes aux modèles théoriques prévus par la Relativité d'échelle de L. Nottale [1, 2] ou peuvent être établis dans le cadre de la RE lorsque ceux-ci ont été d'abord empiriquement établis, alors nous pourrons considérer que la géomorphologie théorique sur une base géométrique est fondée et que c'est dans ce sens préférentiellement que les géographes qui ont en particulier l'analyse spatiale en partage doivent travailler que leurs 


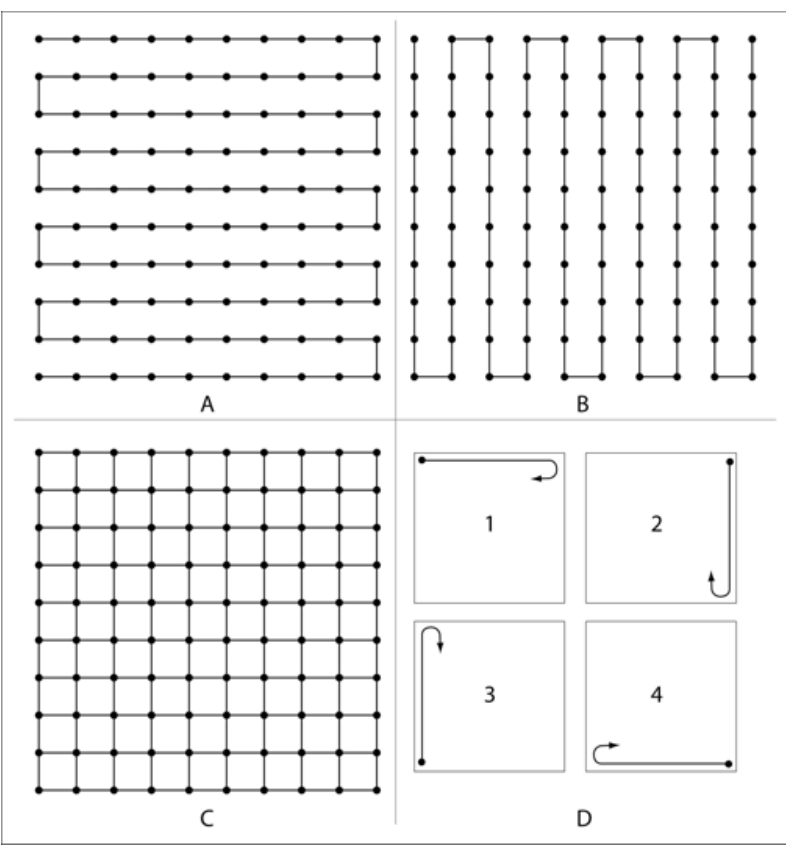

Fig. 1. Exemples de modalités de déploiement des Coupes cordées Topographiques et sens de ces dépliements : A et B : $\mathrm{CcT}^{1}, \mathrm{C}$ : $\mathrm{CcT}^{2}, \mathrm{D}$ : sens des dépliements possibles, $1:$ d'ouest en est puis du nord au sud, etc.

objets d'étude soient naturels et/ou anthropiques. Il est clair que ce serait un premier pas permettant de fonder une géographie théorique sur une base déductive, ce qui pourrait avoir une portée considérable.

Pour arriver à commencer à mettre en œuvre un tel programme, nous avons développé différentes méthodologies $[4,6,10,11,14,15]$ dont nous ne retiendrons ici qu'un aspect qui consiste à extraire de façon spécifique des données d'un Modèle Numérique de Terrain (MNT à pas de $50 \mathrm{~m}$ selon une maille carrée) puis à traiter la série de valeurs obtenues afin d'en calculer l'exposant de Hurst dont on sait qu'il est lié à la dimension fractale (pour une coupe : $\mathrm{D}_{\mathrm{F}}=2-\mathrm{H}$ ). La méthode imaginée consiste à transformer une information caractérisant une surface en une information caractérisant une courbe (Fig. 1).

En faisant cela, nous établissons ce que nous avons appelé des Coupes cordées Topographiques (CcT) qui peuvent être simples $\left(\mathrm{CcT}^{1}\right.$; cf. Fig. 1.a et b : un cheminement selon une direction préférentielle) ou doubles (CcT2 ; cf. Fig. 1.c ; un cheminement continu selon une direction préférentielle auquel succède un cheminement continu selon une autre direction préférentielle qui peut être orthogonale, mais aussi oblique et orientée à 45 ou $315^{\circ}$ diagonales). Par ailleurs, il est possible d'imaginer des allers puis des retours selon le même cheminement ce que nous appelons coupe en miroir $\left({ }^{\mathrm{M}} \mathrm{CcT}\right)$. Il est clair qu'il y a bien d'autres façons théoriques de réordonner l'information disponible dans un MNT et que celles-ci devront être explorées tout comme la combinaison presque infinie des types d'extraction, ceci afin de mesurer quels pourraientêtre les biais introduits par ce type de processus. Il faut noter que les coupes ainsi constituées correspondent à des séries très longues $(+$ de 700000 points pour le Lodévois avec une $\mathrm{CcT}^{1}$, donc plus de 1,4 million avec une $\mathrm{CcT}^{2}$ ou une ${ }^{\mathrm{M}} \mathrm{CcT}$ ), ce qui induit des difficultés de manipulation et des temps de calcul importants.

La méthode la plus rapide et la plus usuelle pour calculer la valeur de $\mathrm{H}$ d'une série de points régulièrement espacés est celle dite R/S. Elle est due à H. E. Hurst [4, 16-19].

Cette statistique, appliquée à nos extractions [4] livre certes la structuration scalaire d'ensemble de l'aire considérée - structuration qui est une transition F-NF - mais sous une forme très bruitée ; bruit que nous ne sommes guère arrivés à filtrer. A l'expérience la méthode qui semble la plus adaptée, car elle livre des résultats extrêmement peu bruités, est celle dite RMS : Roughness - Length Relationship Method [20,21], qui demande toutefois des temps de calcul très importants lorsque les séries sont très longues.

Avec cette méthode, il s'agit, pour une taille de fenêtre donnée (donc pour une échelle spécifique), de calculer la racine carrée de la valeur quadratique moyenne de la «rugosité» du relief considéré. Soit d'après J.-C. Russ [22] (p.54) :

$$
R M S(w)=\frac{1}{N_{w}} \sum_{i=1}^{N_{w}} \sqrt{\frac{1}{m_{i}-2}} \sum_{j \in w_{i}}\left(Z_{j}-\bar{Z}\right)^{2} .
$$

Avec :

- $N_{w}$ est le nombre total de fenêtres de longueur $w$ utilisables dans la série de données ;

- mi est le nombre de points dans la série, corrigé de 2 pour tenir des deux degrés de liberté utilisés pour extraire la tendance ;

- $Z_{j}$ est la valeur après extraction de la tendance ;

- $\bar{Z}$ est la moyenne des valeurs $Z_{J}$ dans chaque fenêtre $w_{i}$.

En d'autres termes, il s'agit de calculer, pour chaque fenêtre de longueur $w$, après soustraction de la tendance locale représentée par la moyenne des altitudes -, le RMS (RootMean-Square) roughness $(\mathrm{S}(w))$ soit la valeur dite efficace, et d'en faire la moyenne $\langle\mathrm{s}(w)\rangle$. Le logarithme népérien de chacune de ces valeurs est ensuite porté en fonction du logarithme népérien de la taille de la fenêtre $w$ qui joue ici le rôle de jauge. Les calculs ont été faits sous Benoit 1.3 de TruSoft International.

$$
\begin{aligned}
& \text { Et donc : } \\
& \langle\mathrm{s}(w)\rangle=w^{\mathrm{H}} \\
& \mathrm{Et}: \\
& \mathrm{D}_{\mathrm{RMS}}=2-\mathrm{H} ;
\end{aligned}
$$

$\mathrm{D}_{\mathrm{RMS}}$ étant la dimension fractale de la série calculée avec la méthode RMS

Toutefois, là où la méthode $\mathrm{R} / \mathrm{S}$ n'introduit et n'utilise que des valeurs minimales et maximales des écarts à la moyenne de la série dont l'écart est normé par la variance, la méthode RMS, elle, considère, après extraction d'une tendance, des écarts à une valeur fixe (la moyenne des $Z$ de la fenêtre). Cela revient à dire qu'une élévation par exemple de $10 \mathrm{~m}$ en moyenne d'une fenêtre à la suivante ne peut pas apparaître, à ce niveau, dans les calculs. C'est en cela que la variation en tendance, de fenêtre en fenêtre de taille semblable, est neutralisée ; ce qui n'est pas le cas avec la méthode R/S. La méthode RMS introduit donc une forme de lissage en ne considérant que des écarts relatifs là où 


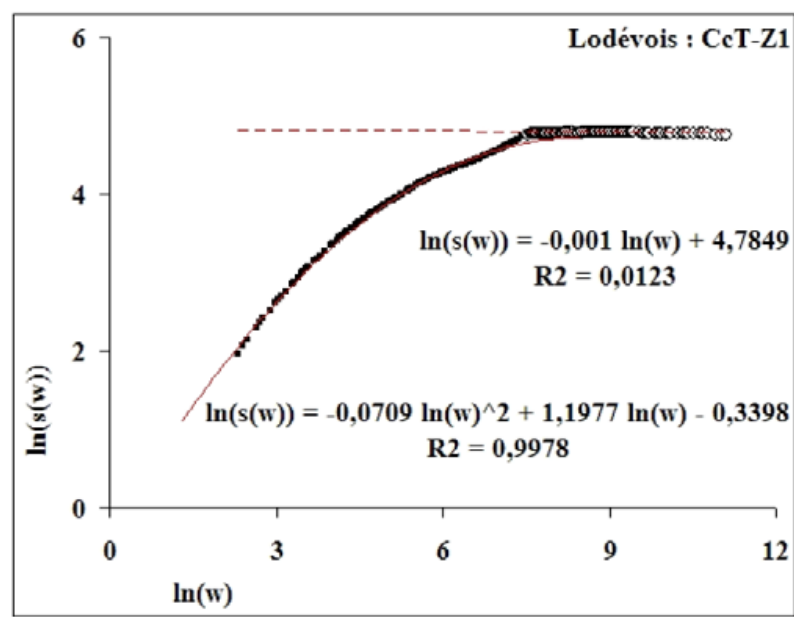

Fig. 2. Statistique RMS sur la CcT-Z1 du MNT du Lodévois.

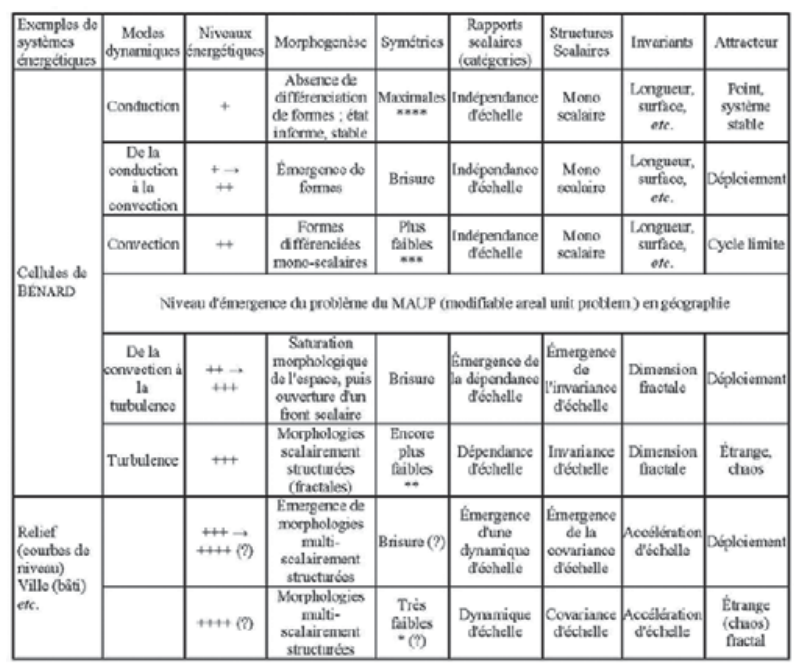

Fig. 3. Typologie des relations entre dynamiques, symétries, structures scalaires et espaces de contrôle (attracteur) d'après Martin [23].

la méthode R/S prend en compte des écarts absolus. Ceci conduit à des résultats infiniment moins bruités.

Le premier calcul a été effectué sur une coupe (Fig. 1.a) dite CcT-Z1. La figure 2 obtenue avec cette CcT-Z1 montre que la relation $\ln (w)$ vs $\ln (\mathrm{s}(w))$ n'est pas rectiligne jusqu'à une échelle voisine $2,71^{8}$ soit environ $145 \mathrm{~km}$ de coupe. Audelà, la valeur de $\ln (\mathrm{s}(w))$ est maximale et constante.

La dimension $\mathrm{D}_{\mathrm{RMS}}$ est donc très voisine de deux sur la gamme d'échelles supérieure à $2,71^{8}$. Par contre la première partie peut être ajustée empiriquement à un polynôme du second degré avec un assez bon coefficient de détermination $\left(\mathrm{r}^{2}=0,998\right)$.

Nous avons donc là d'une part une transition entre une situation fractale (entre la plus grande échelle et $\mathrm{s}(w)=$ $2,71^{8}$ ) et une situation indépendante d'échelle pour les plus petites échelles (au-delà de $\mathrm{s}(w)=2,71^{8}$ ) et d'autre part une portion de gamme scalaire sur laquelle une structuration en échelle ne correspond pas à une invariance d'échelle. Dans ce cas, dans le graphique bilogarithmique les points devraient s'aligner selon une droite. Or, nous observons une courbure continue, ce qui traduit l'existence d'un facteur

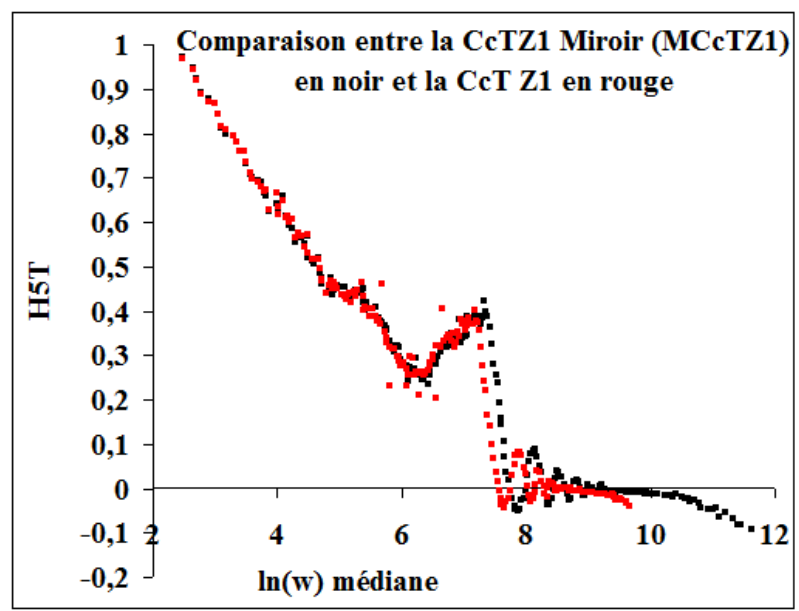

Fig. 4. Tangente locale calculée sur 5 valeurs de couples $\ln (w)$ vs $\ln (\mathrm{s}(w))$ de la CcT-Z1 et de la ${ }^{\mathrm{M}} \mathrm{CcT}-\mathrm{Z} 1 \mathrm{du}$ MNT du Lodévois.

supplémentaire (une force d'échelle dans le vocabulaire de la RE) qui aboutit à une accélération d'échelle (Fig. 3).

Une telle situation était attendue pour des systèmes hautement énergétiques, ce qui est le cas avec le Lodévois entre le Larzac sud et l'Hérault. Toutefois, ce n'est rien comparativement à ce que nous pourrions observer dans les Alpes et a fortiori dans l'Himalaya ; cas qui sont en cours d'exploration.

Cela étant, si nous avions mis en évidence une situation d'invariance d'échelle (dimension fractale constante sur la gamme scalaire considérée), nous aurions été en mesure de dire que nous avions déterminé l'invariant scalaire de ce relief. Or, il n'en est rien puisque nous mettons en évidence une covariance d'échelle (une dimension fractale variable). Pour atteindre l'invariant de cette structuration scalaire, il faut donc dériver la relation établie entre $\ln (w)$ vs $\ln (\mathrm{s}(w))$. Ceci aurait pu être fait formellement. Toutefois, dans une première approche nous avons calculé des tangentes locales à cette courbe sur 5 échelles $\left(\mathrm{H}_{5 \mathrm{~T}}\right)$. Ceci permet d'obtenir des pentes locales qui sont autant de dimensions fractales locales dont il est ensuite possible de voir la variation en fonction des échelles (Fig. 4).

La figure ci-dessus fait apparaître autrement la transition décrite à partir de la figure 2 . Entre les plus grandes échelles $(\ln (w)=2)$ et $\ln (w)=6$ apparait une variation régulière, linéique de la valeur locale de $\mathrm{H}_{5 T}$ donc de la dimension fractale empirique. Au-delà se développe une oscillation qui se calque sur la transition la rendant peut-être possible et qu'il faudra modéliser. Sans cette oscillation, le modèle sous-jacent est connu en RE ([3], Fig. 4.17, 4.20). Cette dernière pourrait s'apparenter en partie à une loi d'échelle non linéaire avec correction log-périodique ([3], Fig. 4.18). Cette oscillation conduit par amortissement successif à une mise à zéro de la valeur expérimentale de $\mathrm{H}_{5 \mathrm{~T}}$. Toutefois il apparait pour de très petites échelles manifestement un biais puisque $\mathrm{H}_{5 \mathrm{~T}}$ devient négatif. Ceci est très certainement dû à l'effectif trop limité des fenêtres de grande taille. Pour accroître cet effectif, il faudrait en théorie augmenter la taille de la surface couverte, mais cela ne ferait que reporter le problème à des échelles encore plus petites.

Pour contourner cette difficulté, nous avons imaginé de doubler l'information sur laquelle se fait le calcul (mais 
on pourrait la tripler, la quadrupler, etc.) tout en gardant le même ordre topologique. Ceci aboutit aux coupes dites en miroir. L'idée est de considérer qu'étant arrivé au terme du cheminement, d'un déploiement (quelle que soit son orientation) il est possible de rebrousser chemin, c'est-àdire revenir au point de départ. Cela revient à créer une CcT symétrie par rapport au dernier point de la $\mathrm{CcT}^{1}$, donc de mettre en œuvre une symétrie en miroir. Pour cette raison, nous appellerons ces $\mathrm{CcT}$ des $\mathrm{CcT}$ Miroir que nous coderons : ${ }^{\mathrm{M}} \mathrm{CcT}$. Donc, la longueur d'un ${ }^{\mathrm{M}} \mathrm{CcT}$ est égale à deux fois celle d'une $\mathrm{CcT}^{1}$. En multipliant ainsi la longueur sur laquelle les calculs peuvent être faits, il apparaît clairement que les déterminations sur les grandes tailles de fenêtres qui correspondent à peu de valeurs sur lesquelles une moyenne peut être effectuée, sont améliorées puisque la moyenne obtenue s'appuiera sur bien plus de valeurs expérimentales. De même, on peut espérer, pour toutes les tailles de fenêtre, une baisse du bruit, donc un signal plus clair et plus précis. La difficulté est par contre que les temps de calcul sont très longs (environ une vingtaine d'heures pour 1400000 points avec un PC standard).

Un premier essai a été effectué avec la CcT-Z1 dupliquée en miroir ( $\left.{ }^{\mathrm{M}} \mathrm{CcT}-\mathrm{Z} 1\right)$ à partir de laquelle il est possible de calculer des valeurs locales de $\mathrm{H}_{5 \mathrm{~T}}$ en déterminant une pente scalairement locale sur 5 fenêtres. Le résultat en est donné dans la figure 3 . Très clairement, les fluctuations sont semblables à celles obtenues avec la CcT-Z1. Il est donc possible de superposer les deux distributions de points pour mieux mesurer les écarts. Il apparait aussi que la structure d'ensemble est strictement la même : transition entre une situation fractale aux grandes et moyennes échelles et une situation non fractale aux petites et très petites échelles et que les oscillations sont aussi bien présentes. Par contre, elles sont un peu décalées dans les échelles comme si la limite induite par la longueur de la CcT-Z1 ne pesait plus (ou moins) sur le calcul fait avec la ${ }^{\mathrm{M}} \mathrm{CcT}$. On notera aussi avec satisfaction que le biais des très petites échelles est repoussé très loin et que l'indépendance d'échelle est très bien établie et est très stable au moins jusqu'à $\ln (w)=10$ ce qui représente presque $1100 \mathrm{~km}$. De même, le bruit (les écarts pour certains points à la tendance locale globale) est réduit. Nous avons donc là un signal expérimental qui peut donner lieu à un développement déductif et à l'ajustement d'un modèle analytique, constituant ainsi un objet de connaissance. Cela fait nous accèderions donc alors à la structure du générateur théorique dont l'expression peut conduire à ce résultat morphologique qui n'est pas dénué quand nous sommes sur le terrain d'une certaine esthétique. Il ne resterait plus alors qu'à déterminer le plan de variabilité d'une telle structure.

Évidemment, il faut explorer d'autres directions de dépliement (cas B et $\mathrm{C}$ de la Figure 1), d'autres points de départ (Fig.1D), d'autres combinaisons en miroir, etc. Nous avons commencé à le faire, mais il est impossible dans ce texte d'évoquer tous les résultats obtenus. Par contre, il est possible de tracer des perspectives assez générales.

Deux voies non exclusives s'ouvrent en fait à nous :

- d'une part, remonter théoriquement au générateur, le caractériser et en calculer la traduction archétypale ; c'est-à-dire son expression morphologique fondamentale, soit le relief type ;

- d'autre part, explorer d'autres espaces, d'autres morphologies : ici un plateau, là une montagne avec ou sans traces fortes d'érosion glaciaire, etc. afin de voir quelle est la gamme des générateurs qui sont en arrière-plan des morphologies et évidemment essayer de les mettre en rapport avec les désignations phénoménologiques.

Il restera ensuite (ou concomitamment) à appliquer tout cela à d'autres entités géographiques. On peut penser à une canopée, à la morphologie du bâti des villes, etc. pour voir si toutes ces morphologies perçues différemment ne se résument pas à quelques cas de structures scalaires ; pari que nous faisons volontiers.

\section{Références}

1. Nottale L., 1993, Fractal space-time and microphysics. Towards a theory of scale relativity. Word Scientific Publishing, London, 333 p.

2. Nottale L., 2011, Scale Relativity and Fractal SpaceTime: A New Approach to Unifying Relativity and Quantum Mechanics. Imperial College Press, 750 p. http://physio-geo.revues.org/2169

3. Martin Ph., 2012a, Développements scalaires plus ou moins amples aux différentes altitudes du bassin versant du Gardon (Gard, France). Recherche de la grande échelle de coupure portant localement la transition fractal - non fractal. Rencontres d'Avignon (2007 - 2009) autour de la relativité d'échelle, sous la direction de L. Nottale et $\mathrm{Ph}$. Martin, Actes numériques, Collection ESPACE - ISBN : 2-910545-07-5, p.147152.

4. Martin Ph., 2012b, Pour une théorie analytique et géométrique des formes en géographie. Dépendance (ou indépendance) d'échelle en géomorphologie, Rencontres d'Avignon (2007-2009) autour de la relativité d'échelle, sous la direction de Laurent Nottale et Philippe Martin, Actes numériques, Collection ESPACE - ISBN : 2-910545-07-5, p.153-259.

5. Martin Ph., Forriez M., Nottale L., 2012, Le fil d'Ariane du dédale scalaire de l'espace-temps géographique. Géopoint $2010 n^{\circ}$ 18, Université d'Avignon - UMR ESPACE éditeurs, $30 \mathrm{p}$.

6. Nottale L. Martin Ph. Forriez M., 2012, Analyse en relativité d'échelle du bassin versant du Gardon (Gard, France). Étude de la variabilité de la dimension fractale en fonction de l'altitude et de l'échelle, Revue Internationale de Géomatique, p.103-133.

7. Mandelbrot B., 1967, How long is the coast of Britain? Statistical self - similarity and fractional dimension. In: Classics on fractals, edited by G.E. Edgar, 1993, Addison - Wesley publishing company, Reading, Massachusetts, USA, p.351-358.

8. Mandelbrot B., 1975, Les objets fractals, 1re édition, Flammarion éditeur, Paris, et 1984, 2e éd., Flammarion 203 p. et 1995, $4^{\text {ème }}$ éd., Flammarion, coll.: Champ $\mathrm{n}^{\circ} 301,208 \mathrm{p}$. 
9. Martin Ph., 2010, L'optimisation en géographie est-elle une optimisation spatiale donc formelle et scalaire ? dans : Optimisation de l'espace géographique et satisfactions sociétales. Avignon, Géopoint 2008 $n^{\circ}$ 17, Groupe Dupont et UMR ESPACE 6012 du CNRS éditeurs, p.185-194.

10. Martin Ph., 2000a, Quelle est la dimension du massif karstique de la Sainte Baume ? Éléments pour une théorie spatiale et fractale du karst. Karstologia, $\mathrm{n}^{\circ}$ 35, p.13-26. http://www.geo.univavignon.fr/Personnel/MartinP/Karstologia\%20n\%C2\% B035.pdf

11. Martin Ph., 2000b, Forme et rugosité des surfaces karstiques. Conséquences pour une théorie spatiale et fractale de l'interface terrestre. Karstologia, $\mathrm{n}^{\circ} 36$, p.1-16. http://www.geo.univ-avignon.fr/Personnel/ MartinP/Karstologie\%20n\%C2\%B036.pdf

12. Martin Ph., 2004, Modélisation fractale et structurelle des formes en géographie. Réflexion développée à partir d'exemples karstiques. Habilitation à diriger les recherches. Université d'Avignon et des Pays du Vaucluse, tome 1, 173 p., tome 2, 314 p., tome 3, 176 p., 1 carte coul. ht. http:/ / www.geo.univavignon.fr / Personnel / MartinP / MartinP-HDR2004 Avignon. pdf

13. Wehbi D., 1986, Approche fractale de la rugosité des surfaces et implications analytiques. Thèse d'État, Université de Franche-Comté, Besançon, 209 p.

14. Martin Ph., 2007, Caractérisation fractale du relief du Lodévois après extraction de tendances topographiques générales. Revue internationale de géomatique. Informations géographiques. Structuration, extraction et utilisation, sous la direction de $\mathrm{Ch}$. Weber et $\mathrm{P}$. Gançarski, Lavoisier - Hermes éditeur, vol. 17, n 3-4, Paris, p.391-414.
15. Martin Ph., 2008, Approche fractale des Grands Causses (Massif Central, France). Structure spatiale de la relation existant entre l'altitude et la dimension fractale de courbes de niveau covariantes. Actes des Journées de l'Association Française de Karstologie de Sion (Suisse), Université de Lausanne organisateur, Coll. EDYTEM - Cahiers de Géographie, n 7, p.93108.

16. Hurst H.E., 1954, Le Nil. Description générale du fleuve; utilisation de ses eaux. Payot éditeur, Paris, $302 \mathrm{p}$.

17. Hurst H.E., Black R.P., Simaika Y.M., 1965, Long-term storage. An experimental study. Constable, Co. Ltd, London, $145 \mathrm{p}$.

18. Mandelbrot B., 1997, Fractals and scaling in finance. Discontinuity, concentration, risk. Springer, Berlin, $551 \mathrm{p}$.

19. Beran J., 1994, Statistics for long-memory processes. Chapman, Hall editors, New York, $315 \mathrm{p}$.

20. Mainsah E., Greenwood J.A., Chetwynd D.G., 2001, (sous la direction de) Metrology and properties of engineering surfaces. Springer - Verlag, New York, $476 \mathrm{p}$.

21. Barabasi A.L. et Stanley H.E., 2002, Fractal concepts in surface growth. Cambridge university press, $366 \mathrm{p}$.

22. Russ J., 1994, Fractal surfaces, Plenum press, New York, 309 p.

23. Martin Ph., 2011, Géographie fractale : fractals auto-similaire et auto-affine, André Dauphiné, 2011. Physio-Géo [En ligne], Volume 5 - 2011, 12 p., mis en ligne le 29 novembre 2011. URL : http:// physiogeo.revues.org/2169 